\title{
ON BAKER'S FINITE BASIS THEOREM FOR CONGRUENCE DISTRIBUTIVE VARIETIES ${ }^{1}$
}

\author{
STANLEY BURRIS
}

\begin{abstract}
This paper contains the simplest, most direct proof of Baker's Theorem to date together with an easy argument which shows how to find an exponential bound on the number of variables needed in a finite basis.
\end{abstract}

Several years ago Baker [1] announced his now famous theorem that every congruence distributive variety generated by a finite algebra has a finite equational basis. Baker's proof in [2] was based on (i) the well-known results of Jónsson for congruence distributive varieties applied to find (ii) a technique for constructing an (infinite) equational basis for a variety generated by a class of algebras defined by a universal disjunct of equations, and (iii) a Ramsey argument to obtain an explicit finite set of equations. Baker's Theorem generalized the earlier result of McKenzie [8] concerning varieties of lattices with finitely many additional operations. After Baker's result was announced Herrmann [4] discovered a way to shorten the proof for the case of lattices. Subsequently Makkai [7] showed how to circumvent the Ramsey argument by invoking compactness, but he made no claims for finding an explicit finite basis. Recently Jónsson [6] and Taylor [10] gave yet simpler proofs. Taylor also points out that his proof via compactness leads to a recursive procedure for finding a finite basis. However Baker's proof gives an exponential bound on the number of variables needed in an equational basis, hence it is more constructive.

We justify yet another proof of Baker's Theorem on the grounds that we have further reduced the details needed for a (complete) proof of the existence of a finite basis, and because we have obtained an exponential bound on the number of variables needed in an equational basis by a simple direct argument. Our inspiration for this paper came from Baker's survey talk at Oberwolfach during the summer of 1976 . He pointed out that whereas a congruence distributive variety generated by a finite algebra need not have definable principal congruences (Burris [3]), nonetheless the intersection of principal congruences being not equal to the diagonal relation is definable.

Received by the editors November 21, 1977.

AMS (MOS) subject classifications (1970). Primary 08A15, $02 \mathrm{H} 05$.

Key words and phrases. Finite basis theorem, variety, congruence distributive.

'Research supported by NRC Grant \#A7256. 
McKenzie [9] had only recently proved that if a variety has definable principal congruences and (up to isomorphism) a finite set of subdirectly irreducible algebras, all finite, then the variety has a finite equational basis (assuming the language has only finitely many fundamental operations). Our original goal was to generalize McKenzie's result-however we now state that program as a problem: does every variety of finite type with (up to isomorphism) finitely many subdirectly irreducible algebras, all finite, and such that 'the intersection of two principal congruences is not the diagonal relation' is definable have a finite equational basis? In the following we will need the fact that 'the intersection of two principal congruences is not the diagonal relation' is definable by a very special kind of formula called $\delta_{N}$.

The results of this paper were obtained in the Universal Algebra and Model Theory Seminar in Waterloo-special thanks are due to S. BulmanFleming and K. McDowell for their contributions to this subject.

1. The existence of a finite basis. Throughout the first two sections of this paper we are working with algebras of an arbitrary but fixed finite type $\tau$, and a subset $T$ of the fundamental ternary operation symbols. We say that $T$ is adequate for a variety $\mathbf{V}$ (of type $\tau$ ) if

$$
\begin{aligned}
& \mathrm{V} \vDash t(x, u, x)=t(x, v, x) \quad \text { for } t \in T, \\
& \mathrm{~V} \vDash x \neq y \rightarrow \underset{t \in T}{\bigvee} t(x, x, y) \neq t(x, y, y) .
\end{aligned}
$$

Define $P$ to be the set of $\tau$-polynomials $p(x, \vec{z})$ such that (i) no variable occurs twice in $p(x, \vec{z})$, and (ii) the variable $x$ occurs in every nonvariable subterm of $p(x, \vec{z}) .^{2}$ If $A$ is an algebra and $\langle a, b\rangle \in A$ then $\theta(a, b)$ will denote the principal congruence generated by $\langle a, b\rangle . \Delta$ denotes the diagonal relation (of whichever algebra is being considered).

Lemma 1.1. Suppose $T$ is adequate for $\mathbf{V}$. Then for $a, b, a^{\prime}, b^{\prime} \in A \in \mathbf{V}$ we have $\theta(a, b) \cap \theta\left(a^{\prime}, b^{\prime}\right) \neq \Delta$ iff

$$
\begin{aligned}
& \underset{\substack{p, q \in P \\
t \in T}}{\bigvee} \exists \vec{z} \exists \vec{w}\left[t\left(p(a, \vec{z}), q\left(a^{\prime}, \vec{w}\right), p(b, \vec{z})\right)\right. \\
&\left.\neq t\left(p(a, \vec{z}), q\left(b^{\prime}, \vec{w}\right), p(b, \vec{z})\right)\right] .
\end{aligned}
$$

Proof. If $\boldsymbol{A}$ satisfies the disjunct of sentences above then choose $\vec{e}, \vec{f}$ from $A$ such that

$$
t\left(p(a, \vec{e}), q\left(a^{\prime}, \vec{f}\right), p(b, \vec{e})\right) \neq t\left(p(a, \vec{e}), q\left(b^{\prime}, \vec{f}\right), p(b, \vec{e})\right) .
$$

From condition (1) it is clear that

$$
\begin{gathered}
\left\langle t\left(p(a, \vec{e}), q\left(a^{\prime}, \vec{f}\right), p(b, \vec{e})\right), t\left(p(a, \vec{e}), q\left(b^{\prime}, \vec{f}\right), p(b, \vec{e})\right)\right\rangle \\
\in \theta(a, b) \cap \theta\left(a^{\prime}, b^{\prime}\right),
\end{gathered}
$$

\footnotetext{
${ }^{2}$ This corresponds to the SAF's of Taylor [10]. Evelyn Nelson pointed out that the definition of $P$ given in a previous draft was inadequate.
} 
hence $\theta(a, b) \cap \theta\left(a^{\prime}, b^{\prime}\right) \neq \Delta$. Conversely, suppose

$$
\theta(a, b) \cap \theta\left(a^{\prime}, b^{\prime}\right) \neq \Delta \text {, say } c \neq d \text { and }\langle c, d\rangle \in \theta(a, b) \cap \theta\left(a^{\prime}, b^{\prime}\right) \text {. }
$$

Claim. For some $\hat{p} \in P, \hat{t} \in T$ and $\vec{g}$ from $A$ we have

$$
\hat{t}(c, \hat{p}(a, \vec{g}), d) \neq \hat{t}(c, \hat{p}(b, \vec{g}), d) .
$$

To see this first note that the equivalence relation on $A$ generated by $\{\langle\hat{p}(a, \vec{g})$, $\hat{p}(b, \vec{g})\rangle \mid \hat{p} \in P, \vec{g}$ from $A\}$ is $\theta(a, b)$ (this is essentially due to $\left.\mathrm{Mal}^{\prime} c e v\right)$. If we always have $\hat{t}(c, \hat{p}(a, \vec{g}), d)=\hat{t}(c, \hat{p}(b, \vec{g}), d), \hat{t} \in T, \hat{p} \in P$ and $\vec{g}$ from $A$, then, as $\langle c, d\rangle \in \theta(a, b), \hat{t}(c, c, d)=\hat{t}(c, d, d)$ for $\hat{t} \in T$, contradicting (2). This establishes the claim, so choose such a $\hat{p}, \hat{t}$ and $\vec{g}$. By incorporating $c, d$ into the parameters we have a $p \in P$ and $\vec{e}$ such that $p(a, \vec{e}) \neq p(b, \vec{e})$, and furthermore $\langle p(a, \vec{e}), p(b, \vec{e})\rangle \in \theta\left(a^{\prime}, b^{\prime}\right)$ as it is in $\theta(c, d)$. But then we can repeat the claim by finding $q \in P, t \in T$, and $\vec{f}$ from $A$ such that

$$
t\left(p(a, \vec{e}), q\left(a^{\prime}, \vec{f}\right), p(b, \vec{e})\right) \neq t\left(p(a, \vec{e}), q\left(b^{\prime}, \vec{f}\right), p(b, \vec{e})\right),
$$

as desired.

For $p \in P$ let us define the depth $d(p)$ of $p$ to be the number of fundamental operation symbols in $p$. Then for $n<\omega$ let $\hat{\delta}_{n}(x, y, u, v)$ be the disjunct

$$
\begin{aligned}
\bigvee_{\substack{t \in \bigvee_{p \in P} \\
d(p), d(q)<n}} \exists \vec{z} \exists \vec{w}[t(p(x, \vec{z}), q(u, \vec{w}), p(y, \vec{z})) & \\
& \neq t(p(x, \vec{z}), q(v, \vec{w}), p(y, \vec{z}))] .
\end{aligned}
$$

Clearly $\hat{\delta}_{n}$ is equivalent to a first-order formula, say $\delta_{n}$.

A simply restatement of Lemma 1.1 is given by the following.

LEMMA 1.2. If $T$ is adequate for $\mathbf{V}$ and $a, b, a^{\prime}, b^{\prime} \in A \in \mathbf{V}$ then $\theta(a, b) \cap$ $\theta\left(a^{\prime}, b^{\prime}\right) \neq \Delta$ iff $A \vDash \bigvee \bigvee_{n<\omega} \delta_{n}\left(a, b, a^{\prime}, b^{\prime}\right)$.

Define $\delta_{n}^{*}$ to be $\forall x \forall y \forall u \forall v\left[\delta_{n+1}(x, y, u, v) \rightarrow \delta_{n}(x, y, u, v)\right]$.

LEMMA 1.3. Let $T$ be adequate for $\mathrm{V}$. Then $\mathrm{V} F \delta_{n}^{*} \rightarrow \delta_{n+1}^{*}$ and

$$
\mathrm{V} \vDash \delta_{n}^{*} \rightarrow\left[\left(\bigvee_{k<\omega} \delta_{k}(x, y, u, v)\right) \rightarrow \delta_{n}(x, y, u, v)\right], \quad n<\omega
$$

Proof. For the first claim suppose $A \vDash \delta_{n}^{*}$, and for some $a, b, c, d \in A$, $A \vDash \delta_{n+2}(a, b, c, d)$. We want to show that $A \vDash \delta_{n+1}(a, b, c, d)$. Choose $t \in T$, $p, q \in P$ with $d(p), d(q) \leqslant n+2$, and $\vec{f}, \vec{g}$ from $A$ such that

$$
t(p(a, \vec{f}), q(c, \vec{g}), p(b, \vec{f})) \neq t(p(a, \vec{f}), q(d, \vec{g}), p(b, \vec{f})) .
$$

Then one can find $p^{\prime}, p^{\prime \prime}, q^{\prime}, q^{\prime \prime} \in P$ such that $d\left(p^{\prime \prime}\right), d\left(q^{\prime \prime}\right)<n+1, d\left(p^{\prime}\right)$, $d\left(q^{\prime}\right)<1$ and

$$
p(x, z)=p^{\prime \prime}\left(p^{\prime}\left(x, \vec{z}_{1}\right), \vec{z}_{2}\right), q(x, \vec{w})=q^{\prime \prime}\left(q^{\prime}\left(x, \vec{w}_{1}\right), \vec{w}_{2}\right) .
$$


Let $a^{\prime}=p^{\prime}\left(a, \vec{f}_{1}\right), b^{\prime}=p^{\prime}\left(b, \vec{f}_{1}\right), c^{\prime}=q^{\prime}\left(c, \vec{g}_{1}\right)$, and $d^{\prime}=q^{\prime}\left(d, \vec{g}_{1}\right)$. Then

$$
\begin{aligned}
t\left(p^{\prime \prime}\left(a^{\prime}, \vec{f}_{2}\right),\right. & \left.q^{\prime \prime}\left(c^{\prime}, \vec{g}_{2}\right), p^{\prime \prime}\left(b^{\prime}, \vec{f}_{2}\right)\right) \\
& \neq t\left(p^{\prime \prime}\left(a^{\prime}, \vec{f}_{2}\right), q^{\prime \prime}\left(d^{\prime}, \vec{g}_{2}\right), p^{\prime \prime}\left(\vec{b}^{\prime}, \vec{f}_{2}\right)\right),
\end{aligned}
$$

hence $A \vDash \delta_{n+1}\left(a^{\prime}, b^{\prime}, c^{\prime}, d^{\prime}\right)$. As $A \vDash \delta_{n}^{*}$ it follows that $A \vDash \delta_{n}\left(a^{\prime}, b^{\prime}, c^{\prime}, d^{\prime}\right)$, so there is a $\hat{t} \in T, \hat{p}, q \in P$ with $d(\hat{p}), d(\hat{q}) \leqslant n$, and $\vec{h}, \vec{k}$ from $A$ such that

$$
\hat{t}\left(\hat{p}\left(a^{\prime}, \vec{h}\right), \hat{q}\left(c^{\prime}, \vec{k}\right), \hat{p}\left(b^{\prime}, \vec{h}\right)\right) \neq \hat{t}\left(\hat{p}\left(a^{\prime}, \vec{h}\right), \hat{q}\left(d^{\prime}, \vec{k}\right), \hat{p}\left(b^{\prime}, \vec{h}\right)\right),
$$

i.e.

$$
\begin{aligned}
\hat{t}\left(\hat{p}\left(p^{\prime}\left(a, \vec{f}_{1}\right), \vec{h}\right), \hat{q}\left(q^{\prime}\left(c, \vec{g}_{1}\right), \vec{k}\right), \hat{p}\left(p^{\prime}\left(b, \vec{f}_{1}\right), \vec{h}\right)\right) \\
\neq \hat{t}\left(\hat{p}\left(p^{\prime}\left(a, \vec{f}_{1}\right), \vec{h}\right), \hat{q}\left(q^{\prime}\left(d, \vec{g}_{1}\right), \vec{k}\right), \hat{p}\left(p^{\prime}\left(b, \vec{f}_{1}\right), \vec{h}\right)\right) .
\end{aligned}
$$

Now $d\left(p\left(p^{\prime}\left(x, \vec{z}_{1}\right), \vec{u}\right)\right)<n+1$ and $d\left(q\left(q^{\prime}\left(x, \vec{w}_{1}\right), \vec{v}\right)\right)<n+1$, so $A$ F $\delta_{n+1}(a, b, c, d)$, as was to be shown. The second claim now follows easily by noting that $\mathbf{V} \vDash \delta_{k} \rightarrow \delta_{k+1}, k<\omega$.

An algebra $A$ is finitely subdirectly irreducible if for $a, b, a^{\prime}, b^{\prime} \in A$ with $a \neq b, a^{\prime} \neq b^{\prime}$ we always have $\theta(a, b) \cap \theta\left(a^{\prime}, b^{\prime}\right) \neq \Delta$. If $\mathbf{V}$ is a variety then $\mathbf{V}_{\mathrm{FSI}}$ denotes the class of finitely subdirectly irreducible algebras in $\mathbf{V}$. A class $\mathbf{K}$ is a basic elementary class if there is a sentence $\varphi$ such that $\mathbf{K}$ is the class of all models of $\varphi$.

LEMMA $1.4 .^{3}$ If $T$ is adequate for $\mathbf{V}$ and $\mathbf{V}_{\mathrm{FSI}}$ is a basic elementary class then, for some $N<\omega$,

$$
\mathbf{V}_{\mathrm{FSI}} \vDash(x \neq y \& u \neq v) \rightarrow \delta_{N}(x, y, u, v)
$$

and $\mathrm{V} \vDash \delta_{N}^{*}$.

Proof. The first claim is just an application of compactness as Lemma 1.2 implies $\mathbf{V}_{\mathrm{FSI}} \vDash(x \neq y \& u \neq v) \rightarrow \bigvee \bigvee_{n<\omega} \delta_{n}(x, y, u, v)$. The second claim uses Lemma 1.2 and the first claim to show $\mathbf{V}_{\mathrm{FSI}} \vDash \delta_{N}^{*}$, and then a direct argument to show that a sentence of the form $\delta_{N}^{*}$ is preserved by subdirect products, hence $\mathbf{V} \vDash \delta_{N}^{*}$.

LEMMA 1.5. Let $T$ be adequate for $\mathbf{V}$, and suppose $\mathbf{V}_{\mathrm{FSI}}$ is a basic elementary class. Let $\sigma$ be a sentence defining $\mathrm{V}_{\mathrm{FSI}}$, let $\mu$ be the sentence

$$
\begin{aligned}
& \forall x \forall u \forall v\left[\&_{t \in T} t(x, u, x)\right.=t(x, v, x)] \\
& \& \forall x \forall y\left[x \neq y \rightarrow \bigvee_{t \in T} t(x, x, y) \neq t(x, y, y)\right],
\end{aligned}
$$

and let $\varepsilon$ be $\forall x \forall y \forall u \forall v\left[(x \neq y \& u \neq v) \rightarrow \delta_{N}(x, y, u, v)\right]$, where $N$ is as in Lemma 1.4. Then $\mathrm{V} \vDash \mu \& \delta_{N}^{*} \&(\varepsilon \rightarrow \sigma)$.

\footnotetext{
${ }^{3}$ The importance of $V_{F S I}$ being a basic elementary class is first pointed out in Jónsson [ 6 .
} 
Proof. $V \vDash \mu$ as $T$ is adequate for $V, V \vDash \delta_{N}^{*}$ by Lemma 1.4, and $V \vDash \varepsilon \rightarrow \sigma$ by Lemma 1.2.

THEOREM 1.6. Suppose $T$ is adequate for $\mathbf{V}$ and $\mathbf{V}_{\mathrm{FSI}}$ is a basic elementary class. Choose $N, \sigma, \mu, \varepsilon$ as above. Then there is a finite set $\Sigma^{*}$ of identities such that $\mathrm{V} \vDash \Sigma^{*}$ and $\Sigma^{*} \vdash \mu \& \delta_{N}^{*} \&(\varepsilon \rightarrow \sigma)$. Any such $\Sigma^{*}$ will be an equational basis for the equational theory of $\mathbf{V}$. Moreover there is a recursive procedure such that, given $\sigma$, we can find such a basis $\Sigma^{*}$.

Proof. Let $\Sigma$ be the set of equations true of V. From Lemma 1.5 $\Sigma \vdash \mu \& \delta_{N}^{*} \&(\varepsilon \rightarrow \sigma)$, so by compactness there must be a finite subset $\Sigma^{*}$ of $\Sigma$ such that $\Sigma^{*} \vdash \mu \& \delta_{N}^{*} \&(\varepsilon \rightarrow \sigma)$. Let $V^{*}$ be the variety defined by $\Sigma^{*}$. To prove that $\mathbf{V}=\mathbf{V}^{*}$ it suffices to show that $\left(\mathbf{V}^{*}\right)_{\mathrm{FSI}} \subseteq \mathbf{V}_{\mathrm{FSI}}$. From $\mathbf{V}^{*} \vDash \mu$ it follows that $T$ is adequate for $\mathrm{V}^{*}$, so suppose $A \in\left(\mathrm{V}^{*}\right)_{\mathrm{FSI}}$ and $a, b, a^{\prime}, b^{\prime} \in A$. If $a \neq b$ and $a^{\prime} \neq b^{\prime}$ then from Lemma $1.2 A \vDash \bigvee_{n<\omega} \delta_{n}\left(a, b, a^{\prime}, b^{\prime}\right)$. As $A \vDash \delta_{N}^{*}$, Lemma 1.3 guarantees that $A \vDash \delta_{N}\left(a, b, a^{\prime}, b^{\prime}\right)$. Thus $A \vDash \varepsilon$, and then from $A \vDash \varepsilon \rightarrow \sigma$ follows $A \vDash \sigma$, i.e. $A \in \mathrm{V}_{\mathrm{FSI}}$. To actually compute such a $\Sigma^{*}$ first enumerate the equational consequences $p_{i}=q_{i}$ of $\sigma$, and then generate (in a parallel process) the consequences of $\Sigma^{(n)}=\left\{p_{i}=q_{i} \mid i \leqslant n\right\}, n<\omega$, until we find, for some $n, \Sigma^{(n)} \vdash \mu, \delta_{N}^{*}$ and $\varepsilon \rightarrow \sigma$. Then let $\Sigma^{*}=\Sigma^{(n)}$.

2. Bounding the number of variables needed in $\Sigma^{*}$. In this section we suppose that $T$ is adequate for $\mathbf{V}$, and $\mathbf{V}_{\mathrm{FSI}}$ is (up to isomorphism) a finite set of finite algebras. Let $\Sigma_{n}, n<\omega$, be the set of $n$-variable identities in $\Sigma$, the identities of $\mathbf{V}$.

LEMMA 2.1 (BIRKHOFF; SEE [2]). One can (constructively) find a finite basis for $\Sigma_{n}, n<\omega$.

LEMMA 2.2. For $n<\omega$ let $A$ be an algebra generated by a set $X$ of at most $n$ elements. Then $A \vDash \Sigma_{n}$ implies $A \in \mathbf{V}$.

Proof. The free algebra on $n$ free-generators in the variety defined by $\Sigma_{n}$ is the same as the free algebra on $n$ free-generators in $\mathbf{V}$.

Let $R$ be the maximum rank of a fundamental operation in $\mathbf{V}$ (note: $R>3$ ), and let $N$ be as in Lemma 1.4 .

LEMMA 2.3. If, for $n<\omega, A \vDash \delta_{n}(a, b, c, d)$ then there is a subalgebra $B$ of $A$ generated by $<2 n(R-1)+4$ elements such that $a, b, c, d \in B$, and we have $B \vDash \delta_{n}(a, b, c, d)$.

Proof. Choose $t \in T, p, q \in P, d(p), d(q) \leqslant n$, and $\vec{f}, \vec{g}$ from $A$ such that

$$
t(p(a, \vec{f}), q(c, \vec{g}), p(b, \vec{f})) \neq t(p(a, \vec{f}), q(d, \vec{g}), p(b, \vec{f})) .
$$

Then let $B$ be the subalgebra generated by $a, b, c, d, \vec{f}$ and $\vec{g}$.

LEMMA 2.4. Let $R^{*}=2(N+1)(R-1)+4$. If $A \vDash \Sigma_{R^{*}}$ then $A \vDash \delta_{N}^{*}$. 
Proof. Suppose $A \vDash \delta_{N+1}(a, b, c, d)$. By Lemma 2.3 we can find a subalgebra $B$ of $A$ generated by $\leqslant R^{*}$ elements and such that $a, b, c, d \in B$ and $B \vDash \delta_{N+1}(a, b, c, d)$. By Lemma $2.2 B \in \mathrm{V}$, hence from Lemma $1.4 B \vDash$ $\delta_{N}(a, b, c, d)$, so $A \vDash \delta_{N}(a, b, c, d)$.

LEMMA 2.5. If $A$ is FSI and $A \vDash \Sigma_{R^{*}}$ then for $n<\omega$ and $a_{1} \neq b_{1}, \ldots, a_{n} \neq$ $b_{n}$ there is a subalgebra $B$ of $A$ generated by $\leqslant(2 n-1)[2 N(R-1)+4]$ elements such that $a_{i}, b_{i} \in B, 1 \leqslant i \leqslant n$, and in $B$ we have $\bigcap_{1<i<n} \theta\left(a_{i}, b_{i}\right) \neq$ $\Delta$.

Proof. As $A \vDash \Sigma_{R^{*}}$ we have $A \vDash \delta_{N}^{*}$ by Lemma 2.4, and as $R^{*} \geqslant 3$ it is clear that $A \vDash \mu$. Hence from Lemmas 1.2 and $1.3 A \vDash \varepsilon$. Now Lemma 2.3 ensures that for $a \neq b$ and $c \neq d, a, b, c, d \in A$, there is a subalgebra $B^{\prime}$ of $A$ generated by $\leqslant 2 N(R-1)+4$ elements such that in $B^{\prime}$ we have $\theta(a, b) \cap$ $\theta(c, d) \neq \Delta$. Applying this to $\left\langle a_{1}, b_{1}, a_{2}, b_{2}\right\rangle,\left\langle a_{3}, b_{3}, a_{4}, b_{4}\right\rangle, \ldots$, we obtain subalgebras $B_{1}^{(1)}, B_{2}^{(1)}, \ldots$ each generated by $\leqslant 2 N(R-1)+4$ elements and such that in $B_{i}^{(1)}$ we have $\theta\left(a_{2 i-1}, b_{2 i-1}\right) \cap \theta\left(a_{2 i}, b_{2 i}\right) \neq \Delta$. So choose $c_{i}^{(1)}, d_{i}^{(1)}$ to be distinct elements in $B_{i}^{(1)}$ such that $\left\langle c_{i}^{(1)}, d_{i}^{(1)}\right\rangle$ is in the intersection of the principal congruences generated by $\left\langle a_{2 i-1}, b_{2 i-1}\right\rangle$ and $\left\langle a_{2 i}, b_{2 i}\right\rangle$ in $B_{i}^{(1)}$. Now repeat this process using the pairs $c_{i}^{(1)}, d_{i}^{(1)}$ (instead of the $a_{i}, b_{i}$ ), where $a_{n}, b_{n}$ is added to the $c_{i}^{(1)}, d_{i}^{(1)}$ pairs in case $n$ is odd. After finitely many repeats we finally end with a single pair $c^{(j)}, d^{(j)}$. The total number of algebras $B_{i}^{(m)}$ considered in this process is $\leqslant 2 n-1$. Let $B$ be the subalgebra of $A$ generated by all the $B_{i}^{(m)}$ 's. Then $B$ is generated by $\left\langle(2 n-1)[2 N(R-1)+4]\right.$ elements, and in $B$ we have $\left\langle c^{(j)}, d^{(j)}\right\rangle \in$ $\cap_{1<i<n} \theta\left(a_{i}, b_{i}\right)$, and $c^{(j)} \neq d^{(j)}$.

THEOREM 2.6. Let $S$ be the maximum size of a member of $\mathbf{V}_{\mathrm{FSI}}$. Then, with $N^{*}=\left[2\left(2_{2}^{S+1}\right)-1\right][2 N(R-1)+4], \Sigma_{N^{*}}$ is a basis for $\Sigma$.

Proof. Suppose $A \vDash \Sigma_{N^{*}}$ and $A$ is FSI. If $A$ has more than $S$ elements choose distinct elements $a_{1}, \ldots, a_{S+1}$. As $N^{*} \geqslant R^{*}$ it follows from Lemma 2.5 that there is a subalgebra $B$ of $A$ generated by $\leqslant N^{*}$ elements such that $a_{1}, \ldots, a_{S+1} \in B$ and further there are two elements $c, d \in B$ such that $c \neq d$ and in $B$ we have $\langle c, d\rangle \in \cap_{1<i<j<s+1} \theta\left(a_{i}, a_{j}\right)$. Let $\theta$ be a maximal congruence of $B$ such that $\langle c, d\rangle \notin \theta$. Then $B / \theta$ is FSI, and as $\left\langle a_{i}, a_{j}\right\rangle \notin \theta$ for $1 \leqslant i<j \leqslant S+1$ we see that $B / \theta$ has cardinality at least $S+1$. However $B / \theta \neq \Sigma_{N^{*}}$, and as $B / \theta$ is generated by $\leqslant N^{*}$ elements we must have $B / \theta \in \mathrm{V}$ by Lemma 2.2. This is a contradiction as $B / \theta$ is too large to be in $\mathbf{V}_{\mathrm{FSI}}$, hence it follows that $A$ has at most $S$ distinct elements. Now, as $S<N^{*}$ we know $A \in \mathrm{V}$ by Lemma 2.2 , so $A \in \mathbf{V}_{\mathrm{FSI}}$.

Finally, it is not difficult to show that one can choose $N \leqslant S^{S}$ (as there are at most $S^{S}$ unary maps on a member of $\mathbf{V}_{\mathrm{FSI}}$ ), giving an explicit bound on the 
number of variables needed in terms of the parameters $R$ and $S$ :

$$
\left[2\left(\begin{array}{c}
S+1 \\
2
\end{array}\right)-1\right]\left[2 S^{S}(R-1)+4\right]
$$

PROBLEM. If a variety has (up to isomorphism) only finitely many subdirectly irreducible algebras, all finite, does it follow that all members of $\mathbf{V}_{\text {FSI }}$ are finite (hence subdirectly irreducible)?

3. Congruence distributive varieties. $\mathbf{V}$ is a congruence distributive variety if for every algebra $A$ in $\mathbf{V}$ the lattice of congruences of $A$ is distributive. The following two lemmas are in [5].

LEMMA 3.1 (JÓNSSON). If $\mathbf{V}$ is a congruence distributive variety then there is a finite sequence $t_{0}, \ldots, t_{k}$ of ternary polynomials such that $\mathbf{V}$ satisfies:

$$
\begin{aligned}
& t_{0}(x, y, z)=x, \quad t_{k}(x, y, z)=z, \\
& t_{i}(x, y, x)=x, \text { for } 0 \leqslant i \leqslant k \\
& t_{i}(x, x, z)=t_{i+1}(x, x, z) \quad \text { if } i \text { is even, } \\
& t_{i}(x, z, z)=t_{i+1}(x, z, z) \quad \text { if } i \text { is odd. }
\end{aligned}
$$

LEMMA 3.2. If $\mathbf{V}$ is a congruence distributive variety generated by a finite algebra $A$ then $B \in \mathbf{V}_{\mathrm{FSI}}$ implies $B$ is a quotient of a subalgebra of $A$.

THEOREM 3.3. Let $\mathbf{V}$ be a congruence distributive variety with a finite number of fundamental operation symbols. (a) If $\mathbf{V}_{\mathrm{FSI}}$ is a basic elementary class defined by $\sigma$ then $\mathrm{V}$ has a finitely based equational theory and there is a recursive procedure to find the finite basis from $\sigma$. (b) If $\mathbf{V}$ is generated by a finite algebra $A$ of size $n$ then the equations $\Sigma$ of $\mathbf{V}$ have as a basis the equations $\Sigma_{M}$ where $M=\left[2\left(\begin{array}{c}n+1 \\ 2\end{array}\right)-1\right]\left[2 n^{n} \cdot(R-1)+4\right]$, where $R=\operatorname{Max}(3$, ranks of the fundamental operations).

Proof. Expand the language of $\mathbf{V}$ by adding new ternary operation symbols $\hat{t}_{i}$ for $0 \leqslant i \leqslant k$ such that $\mathbf{V} \vDash t_{i}(x, y, z)=\hat{t}_{i}(x, y, z), t_{i}$ being a fixed set of polynomials as guaranteed by Lemma 3.1 , and call the new variety $\mathbf{V}^{*}$. Then $\mathbf{V}$ has a finite equational basis iff $\mathbf{V}^{*}$ does, and indeed in the same number of variables. With $T=\left\{\hat{t}_{i} \mid 0 \leqslant i \leqslant k\right\}$ we see by Lemma 3.1 that $T$ is adequate for $V^{*}$. Hence to prove (a) it suffices to note that $\left(V^{*}\right)_{\text {FSI }}$ is a basic elementary class (defined by the same sentence as $\mathbf{V}_{\text {FSI }}$ plus the equations $\left.\hat{t_{i}}(x, y, z)=t_{i}(x, y, z)\right)$ and apply Theorem 1.6. For part (b) we see that Lemma 3.2 guarantees $\mathbf{V}_{\mathrm{FSI}}$ satisfies the hypothesis of $\S 2$, and the $S$ of Theorem 2.6 is bounded by $n$.

ConCluding REMARK. Baker observed that if $T$ is adequate for a variety $\mathbf{V}$ then $\mathbf{V}$ is congruence distributive (hence our Theorem 1.6 does not properly generalize Baker's Theorem). To see this note that in $F_{V}(x, y, z)$ we have, for $t \in T$,

$$
t(x, x, z) \theta(x, z) \cap \theta(x, y) t(x, y, z) \theta(x, z) \cap \theta(y, z) t(x, z, z)
$$


hence

$$
\langle t(x, x, z), t(x, z, z)\rangle \in(\theta(x, z) \cap \theta(x, y)) \vee(\theta(x, z) \cap \theta(y, z))
$$

so by the definition of 'adequate',

$$
\langle x, z\rangle \in(\theta(x, z) \cap \theta(x, y)) \vee(\theta(x, z) \cap \theta(y, z)) .
$$

Now we can continue exactly as in Jónsson [5] to show that $\mathbf{V}$ is congruence distributive.

\section{REFERENCES}

1. K. Baker, Equational bases for finite algebras, Notices Amer. Math. Soc. 19 (1972), p. A-44, Abstract \#691-08-2.

2. __ Finite equational bases for finite algebras in congruence distributive equational classes, Advances in Math. 24 (1977), 207-243.

3. S. Burris, An example concerning definable principal congruences, Algebra Universalis 7 (1977), 403-404.

4. C. Herrmann, Weak (projective) radius and finite equational bases for classes of lattices, Algebra Universalis 3 (1974), 51-58.

5. B. Jónsson, Algebras whose congruence lattices are distributive, Math. Scand. 21 (1967), $110-121$.

6. _ A short proof of Baker's Theorem (preprint).

7. M. Makkai, $A$ proof of Baker's finite basis theorem on equational classes generated by finite elements of congruence-distributive varieties, Algebra Universalis 3 (1973), 174-181.

8. R. McKenzie, Equational bases for lattice theories, Math. Scand. 27 (1970), 24-38.

9. congruences in locally finite varieties, Algebra Universalis 8 (1978), 336-348.

10. W. Taylor, Baker's finite basis theorem, Algebra Universalis 8 (1978), 191-196.

Department of Mathematics, University of Waterloo, Waterloo, Ontario, Canada N2L 3G1 